\title{
Erratum to: Control of endoreduplication of trichome by RPT2a, a subunit of the 19S proteasome in Arabidopsis
}

\author{
Kaori Sako • Yuko Maki · Takashi Aoyama • \\ Derek B. Goto $\cdot$ Junji Yamaguchi
}

Published online: 25 March 2014

(C) The Botanical Society of Japan and Springer Japan 2014

\section{Erratum to: J Plant Res (2010) 123:701-706 \\ DOI 10.1007/s10265-010-0321-x}

In the original publication, the corresponding author had mistakenly included Kumiko K. Imai in the author group. However, the author would like to mention the contribution of Kumiko K. Imai in the acknowledgment.

The revised author group and acknowledgment should appear as below:

Kaori Sako · Yuko Maki · Takashi Aoyama · Derek B. Goto · Junji Yamaguchi

\begin{abstract}
Acknowledgments We are grateful to Drs. Akira Ikeda, Yutaka Sonoda and Ms. Naoko Yamazaki for discussion and technical assistance. We would also like to thank Kumiko K. Imai for providing seeds for research. This work was supported by a grant-in-aid for Scientific Research (nos. 19657013, 19039001, 21025001), in part by the Program for Basic Research Activities for Innovative Bioscience (PROBRAIN) (J.Y.) and grants-in-aid for Scientific Research in a Priority Area (D.B.G.). K.S. acknowledges a Research Fellowships from the Japan Society for the Promotion of Science for Young Scientist (2008-2010).
\end{abstract}

The online version of the original article can be found under doi:10.1007/s10265-010-0321-x.

K. Sako · Y. Maki $\cdot$ J. Yamaguchi $(\bowtie)$

Faculty of Advanced Life Science and Graduate School of Life

Science, Hokkaido University, Kita-ku N10-W8,

Sapporo 060-0810, Japan

e-mail: jjyama@sci.hokudai.ac.jp

K. Sako

e-mail: k-sako@sci.hokudai.ac.jp

Y. Maki

e-mail: y_maki@mail.sci.hokudai.ac.jp

T. Aoyama

Institute for Chemical Research, Kyoto University, Uji, Kyoto,

Japan

e-mail: aoyama@scl.kyoto-u.ac.jp

D. B. Goto

Creative Research Initiative 'Sousei', Hokkaido University,

Sapporo, Japan

e-mail: derekgoto@me.com 